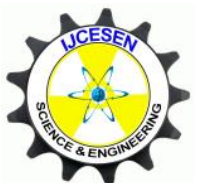

Copyright (C) IJCESEN
International Journal of Computational and

Experimental $\boldsymbol{S}$ cience and $\boldsymbol{E N g i n e e r i n g}$

(IJCESEN)

Vol. 5-No.2 (2019) pp. 80-85

http://dergipark.org.tr/ijcesen

Research Article

\title{
Numerical Simulation Study on the Bottom-Water Invasion in the Carbonate Gas Reservoir with High Angle Fractures and High Permeability Zones
}

\author{
Shuang ZHANG, Huiqing LIU*, Jie BAI
}

State Key Laboratory of Petroleum Resources and Prospecting in China University of Petroleum, Beijing, China.

* Corresponding Author : liuhq110@126.com

ORCID: 0000-0001-7230-5346

\section{Article Info:}

DOI: $10.22399 /$ ijcesen.535713

Received : 05 March 2019

Accepted : 13 July 2019

\section{$\underline{\text { Keywords }}$}

Carbonate gas reservoir

Bottom-water

Pseudo relative permeability

High angle fracture

\begin{abstract}
$\underline{\text { Abstract: }}$
Numerical simulation of carbonate gas reservoir with bottom-water is still a challenging issue, due to the presence of nature conductive fractures. When the water invades into the gas well, the permeability of gas phase decreases sharply. The high angle fractures and high permeability zones have a serious impact on the production performance of the gas wells. The characteristic of the water invasion was unpredictable in conventional dynamic analysis.

In fractured carbonate reservoir, the geometric shapes of the angled fracture are difficult to be described using commercial numerical simulation software. The method that calculating the pseudo relative permeability of the grid block containing high angle fracture was utilized in this paper. Through this method, the rapid water advancement in the conductive fracture was accurately tracked without modifying the grid geometry, and the influence of the conductive fracture to the water breakthrough phenomenon was studied by using the IMEX module in CMG.

The results show that the water invasion intensity depends on the conductivity of fracture, and the higher the conductivity is, the shorter the water breakthrough time is. The breakthrough time of water is affected by the angle of the fracture, the relative horizontal distance, the length of the fracture, the production rate and the type of water invasion. According to the characteristic curve, the type of water invasion can be quickly distinguished, which provides a basis for further study on the enhanced recovery of the fractured carbonate gas reservoir with water.
\end{abstract}

\section{Introduction}

Carbonate gas reservoirs are widely distributed all around the world. The reservoirs generally contain formation water. The strong heterogeneity of the reservoir leads to a complex gas-water relationship [1], and most of the reservoirs are characterized as water channeling [2]. The dramatic decrease of permeability of the gas phase due to water invasion presents a great challenge to the numerical simulation in carbonate bottom water gas reservoirs.

In fractured carbonate gas reservoir with bottomwater, the fracture is the main flow channel [3]. The heterogeneity of the gas reservoir and the irregular movement of the bottom water along the fracture cause the gas reservoir to be divided. The larger the fracture is, the smaller resistance of the bottom water activity is, and the faster the bottom water breakthrough is. When the gas reservoir begins to develop, the pressure wave is transmitted to the water body along the main fracture. As the pressure drop increases, the elastic energy of the water body drives the formation water along the high permeability fracture into the gas zone in a form of "short circuit". For the reservoirs with high storage ratio, the water channeling phenomenon has a great impact to the water breakthrough time [4]. The neglection of the high permeability fracture resulted in the over-prediction problem. Therefore, only by properly characterizing the fracture can we accurately describe the characteristics of the 
fracture water channeling, predict the water breakthrough time of the bottom water, and lay a foundation for the rational workflow.

\section{Methods}

To accurately describe the characteristics of the water channeling in numerical simulation, there are generally two methods: one is local grid refinement, the other is the correction of the relative permeability curves. The second method has better flexibility and availability.

Lingen et al. [5] corrected the relative permeability curves in the grid containing the fractures to evaluate the influence of the fracture to the flow. Ghani et al. [6] adjusted the formula of pseudo permeability curves to rectified the Lingen method. The permeability of grid is calculated as follows:

$$
k_{b}=k_{m}+\frac{k_{f} n_{f} d_{f}}{d_{b}}
$$

where $k_{m}$ is the matrix permeability, $k_{f}$ is the fracture permeability, $d_{b}$ is the grid block size (assumed equal in $\mathrm{x}, \mathrm{y}$ and $\mathrm{z}$ directions), and $\mathrm{n}_{\mathrm{f}}$ is the number of fractures.

The porosity of grid is calculated as follows:

$$
\phi_{b}=\phi_{m}+\frac{\phi_{f} l_{f} d_{f}}{d_{b}^{2}}
$$

where $l_{\mathrm{f}}$ is cumulative fractures length, $\varphi_{\mathrm{f}}$ is the fracture porosity, and $\varphi_{\mathrm{m}}$ is matrix porosity.

In the Lingen [5] method, end points of the curves can be measured for fractures blocks as follows:

$$
\begin{aligned}
k_{r g e, b} & =\frac{k_{g e, m} k_{m} d_{b}+k_{g e, f} k_{f} n_{f} d_{f}}{k_{m} d_{b}+k_{f} n_{f} d_{f}} \\
k_{r w e, b} & =\frac{k_{w e, m} k_{m} d_{b}+k_{w e, f} k_{f} n_{f} d_{f}}{k_{m} d_{b}+k_{f} n_{f} d_{f}}
\end{aligned}
$$

where $\mathrm{k}_{\mathrm{ge}, \mathrm{m}}$ is the end of relative permeability in the matrix, $\mathrm{k}_{\mathrm{ge}, \mathrm{f}}$ is the end of relative permeability to in the fracture, $\mathrm{k}_{\mathrm{we}, \mathrm{m}}$ is the end of relative permeability in the matrix, and $k_{w e, f}$ is the end of relative permeability in the fracture.

In the effective residual saturation are calculated as follows:

$$
\begin{aligned}
& S_{g r, b}=\frac{S_{g r, m} \phi_{m} d_{b}^{2}+S_{g r, f} \phi_{f} l_{f} d_{f}}{\phi_{m} d_{b}^{2}+\phi_{f} l_{f} d_{f}} \\
& S_{w r, b}=\frac{S_{w r, m} \phi_{m} d_{b}^{2}+S_{w r, f} \phi_{f} l_{f} d_{f}}{\phi_{m} d_{b}^{2}+\phi_{f} l_{f} d_{f}}
\end{aligned}
$$

where $S_{\mathrm{gr}, \mathrm{m}}$ is the residual saturation in matrix and $\mathrm{S}_{\mathrm{gr}, \mathrm{f}}$ is the residual saturation in fracture, $S_{\mathrm{wr}, \mathrm{f}}$ is the residual saturation in fracture.

These are the calculations of the normalized curves:

$$
\begin{gathered}
S_{w n, b}=S_{w n, m}\left(1-\alpha_{f}\right)+\alpha_{f} \\
k_{r w, b}^{\prime}=k_{r w, m}+\left(S_{w, b}-k_{r w, m}\right) \beta_{f, w}^{\prime} \\
k_{r g, b}^{\prime}=k_{r g, m}+\left(1-S_{w, b}-k_{r g, m}\right)\left(1-\beta_{m, g}^{\prime}\right) \\
\alpha_{f}=\frac{\left(1-S_{g r, f}-S_{w c, f}\right) l_{f} d_{f} \phi_{f}}{\left(1-S_{g r, f}-S_{w c, f}\right) l_{f} d_{f} \phi_{f}+\left(1-S_{g r, m}-S_{w c, m}\right) d_{b}^{2} \phi_{m}} \\
\beta_{f, w}^{\prime}=\frac{k_{f} k_{r w e, f} n_{f} d_{f}}{k_{f} k_{r w e, f} n_{f} d_{f}+k_{m} k_{r w e, m}\left(d_{b}-n_{f} d_{f}\right)} \\
\beta_{m, g}^{\prime}=\frac{k_{m} k_{r g e, m}\left(d_{b}-n_{f} d_{f}\right)}{k_{f} k_{r g e, f} n_{f} d_{f}+k_{m} k_{r g e, m}\left(d_{b}-n_{f} d_{f}\right)}
\end{gathered}
$$

where $\alpha_{\mathrm{f}}$ is the contribution of the fracture volume to the total mobile porosity in a grid block, $\beta_{f, w}^{\prime}$ is the contribution of fracture to the maximum grid block relative permeability to water, $\beta_{\mathrm{m}, \mathrm{g}}$ is the contribution of the matrix to the maximum grid block relative permeability to gas.

The method of Ghani et al. [6] is applied to one typical relative permeability curve of carbonate gas reservoir. In the Figure 1, four contrast cases are chosen to represent the difference permeability situations, namely, low, medium, high and very high contrast. Based on the analysis, the difference of the permeability between matrix and fracture seriously influenced the pseudo relative permeability curves.
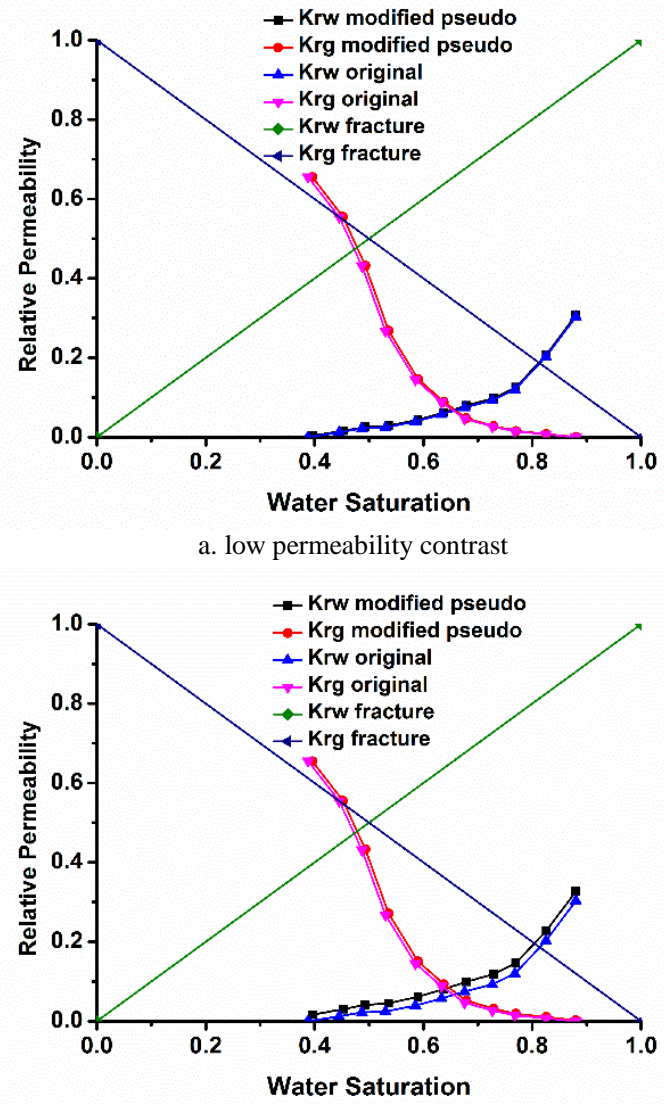

b. medium permeability contrast 


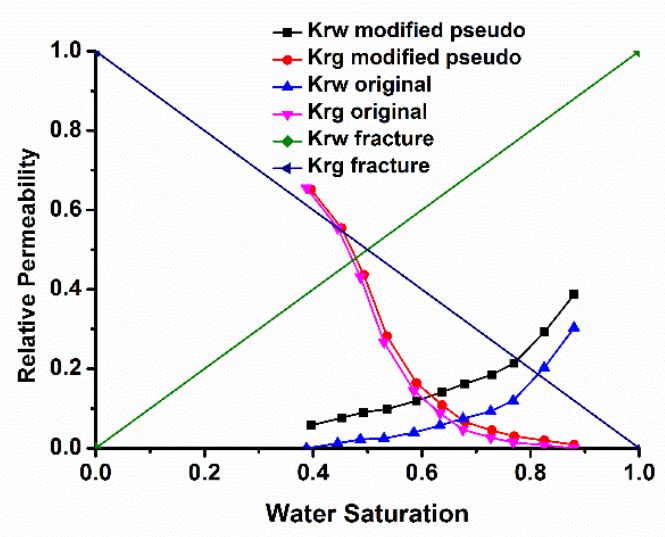

c. high permeability contrast

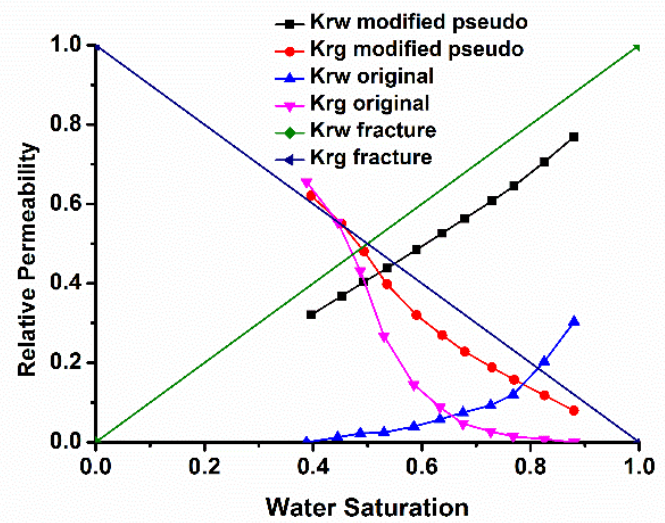

d. very high permeability contrast

Figure 1. Pseudo Relative Permeability Curves Comparison.

In low contrast case (Fig. 1a), there is little change in the matrix and pseudo permeability curves. Therefore, the fluid flows through the matrix and fracture synchronously. In the medium or high cases (Fig. 1b, 1c, 1d), the high permeability has a serious impact on the flow, and leads to the difference between the pseudo permeability and the original matrix permeability curves.

\section{Results and Discussions}

The method was implemented in the realistic model. The model dimensions were $401 \times 401 \times 20$, and the grid size was 2.5 meters.

The effect of fracture angle was shown in Figure 2. When the fracture angle was $90^{\circ}$, the water breakthrough time was the least. The connection between downhole and aquifer was almost straight line. The high permeability fracture provided the convenient channel for the bottom-water, and water flowed into the well very fast. The angle decided the relative distance between the gas-water contact and the well.

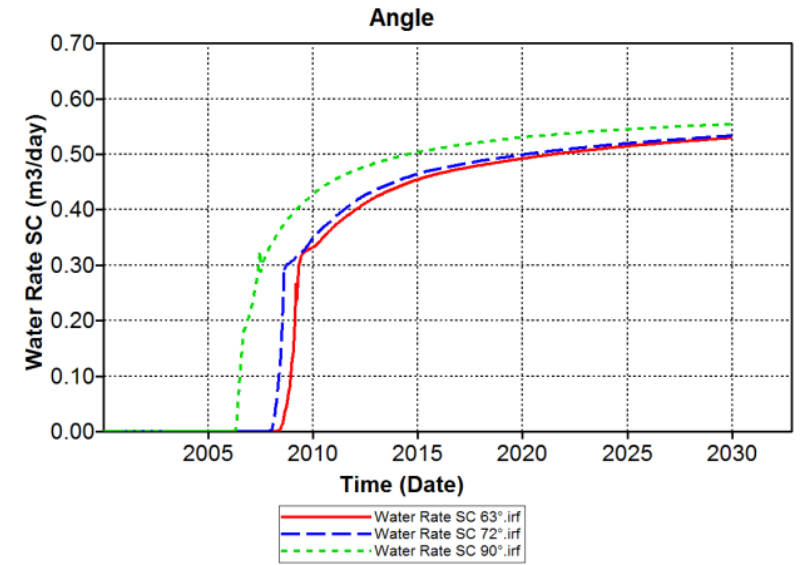

Figure 2. Effect of Angle on Water Rate.

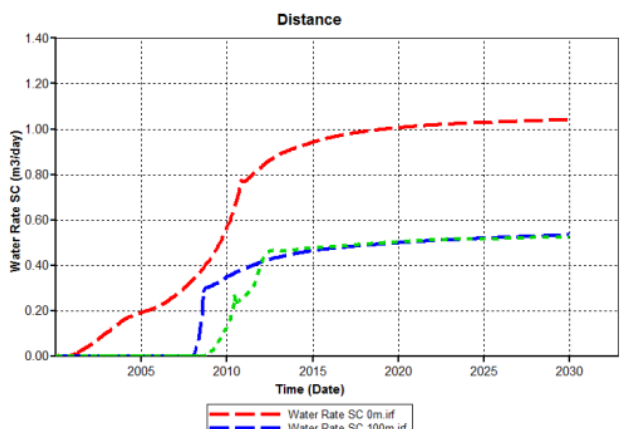

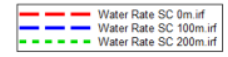

Figure 3. Effect of Distance on Water Rate.

The effect of fracture distance was shown in Figure 3. The fracture horizontal distance from well had a serious impact on the production. The great distance decreased the probability of intersection of the flow region and the fracture. Only the fractures in the pressure funnel influenced the final recovery. The effect of fracture length was shown in Figure 4. The longer the fracture length was, the greater opportunity of encountering the water was.

The effect of the production rate was shown in Figure 5. The major factor of water channeling was the pressure gradient. The high production rate resulted in the fast water invasion. The greater production rate was, the faster pressure wave widened.

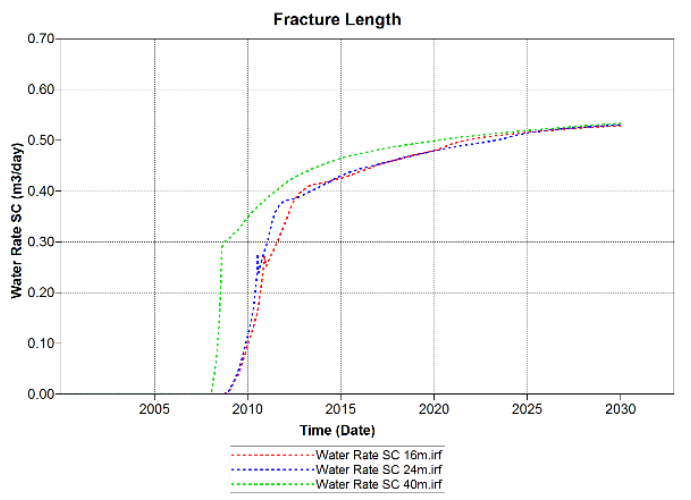

Figure 4. Effect of Length on Water Rate. 


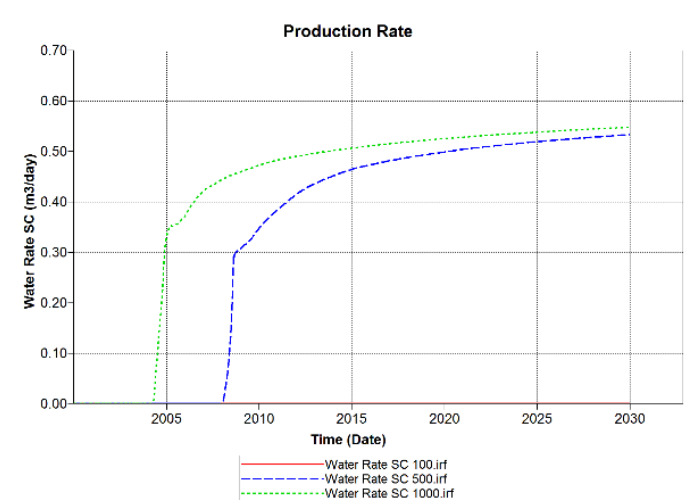

Figure 5. Effect of Production Rate on Water Rate.

Feng et al. [7] summarized four water invasion patterns, namely, the water coning, vertical breakthrough, lateral invasion and compound patterns, as presented in figure 6. This paper introduced the water invasion type into the numerical simulation. The vertical stratigraphic sections of all the types were shown in Figure 7. The red part was the fracture and the high permeability zones. Then, IMEX simulator of CMG was used to investigate the influence of conductive fractures on the water breakthrough phenomenon [8].

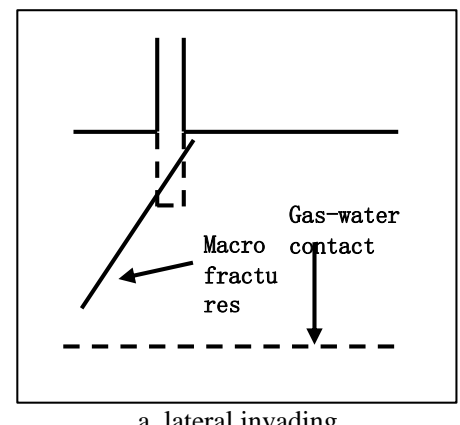

a. lateral invading

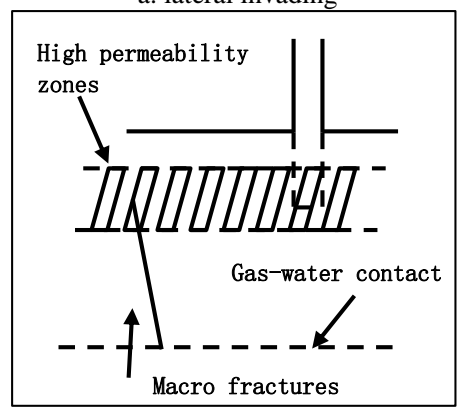

b. vertical channeling-lateral invading

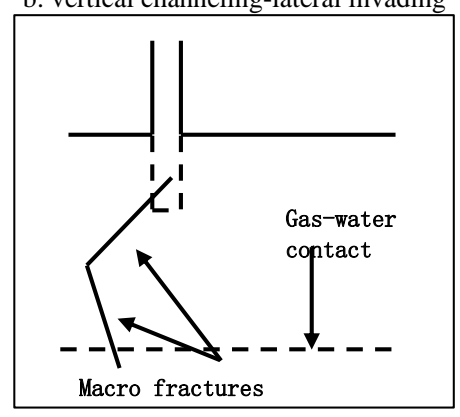

c. vertical channeling

Figure 6. Different Water Invasion Type.
In the period of the production, the water invasion of the vertical channeling-lateral invading type can be tracked by the remarkable saturation in Figure 8 . At the beginning of the opening, the water invaded quickly through the vertical channel and the water saturation around the bottom of the well increased rapidly. It was obvious that in the relative permeability curve (Figure 1), the higher water saturation was, the lower relative permeability of gas was. The characteristics of fracture water breakthrough limited the flow of gas, and enhanced the water activity. The difficulty in removing water block made it worse to resume the gas production.

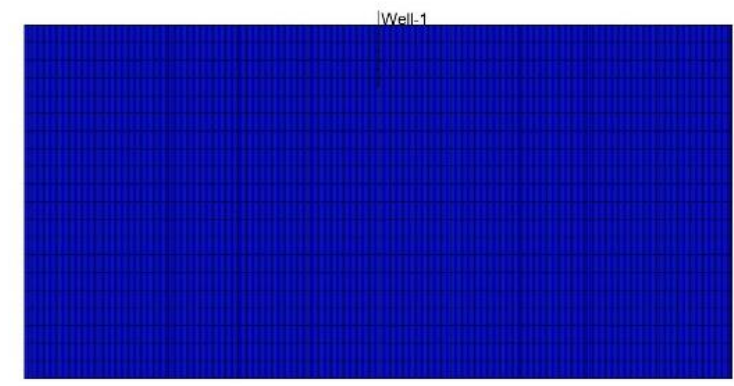

a. water coning

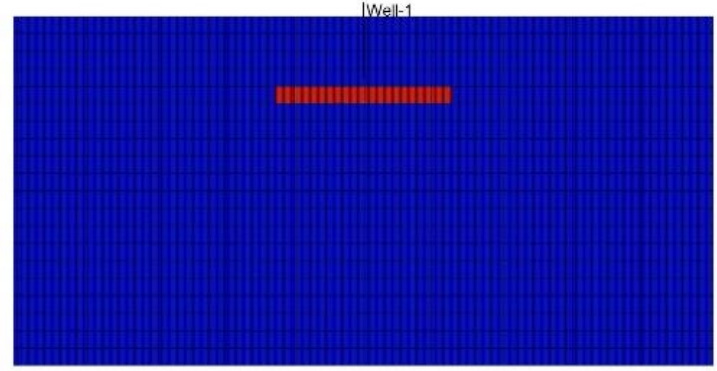

b. lateral invasion

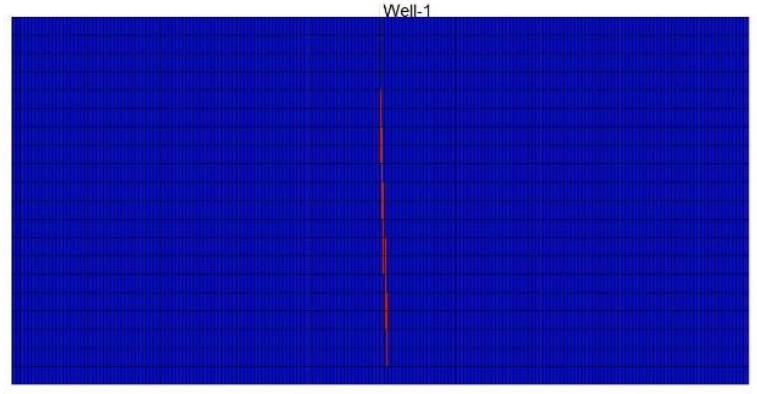

c. vertical channeling

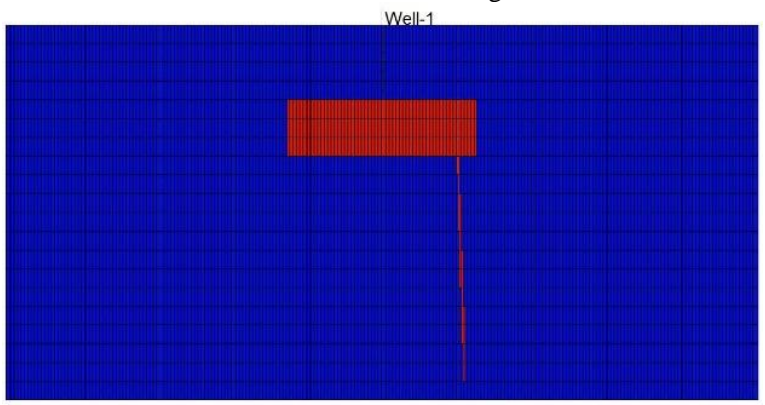

d. vertical channeling-lateral invading

Figure 7. Different Water Invasion Models in IMEX of $C M G$. 


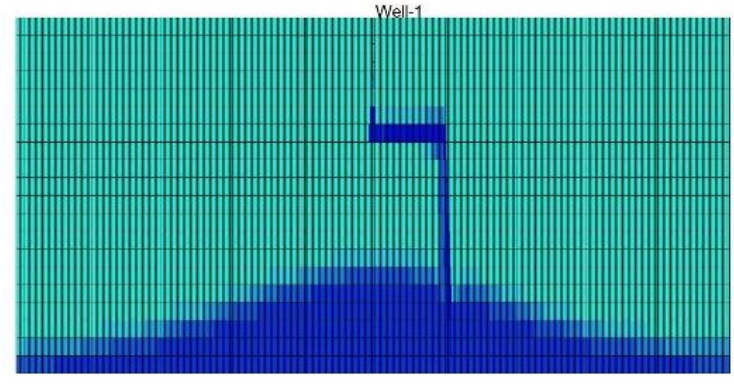

a. time step $=52$

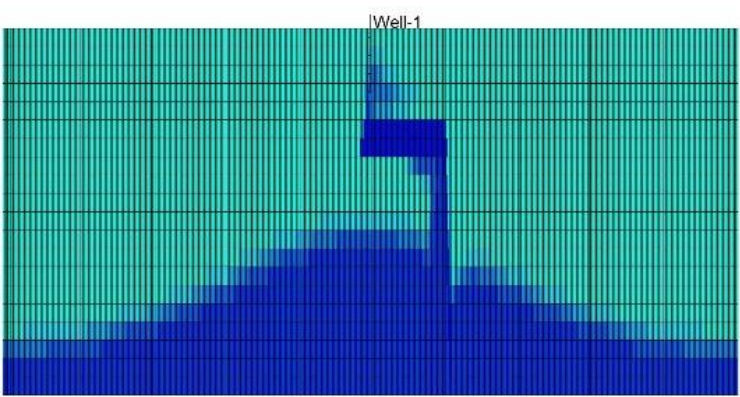

b. time step $=78$

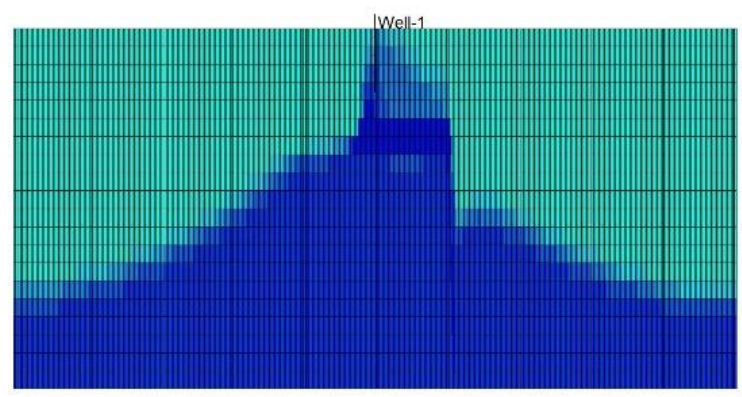

c. time step $=114$

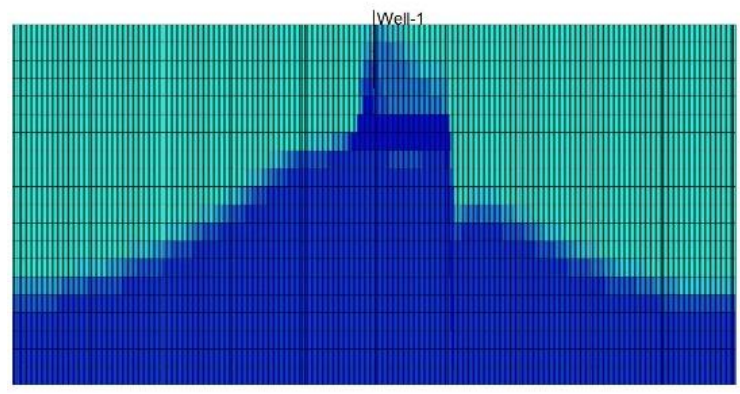

d. time step $=160$

Figure 8. Water Invasion in Different Time Step.

The effect of different invasion type was shown in Figure 9. Even though the water coning make the highest production of water, the focus of this work was water breakthrough time. When the water flowed into the well, no matter how much the amount of water, the destruction was massive. The vertical channeling invasion had the strongest relation to the water breakthrough time. Without the connection of the vertical fracture, some high permeability zones wouldn't do the same harm. The characteristic curves can be established to distinguish the water invasion quickly.

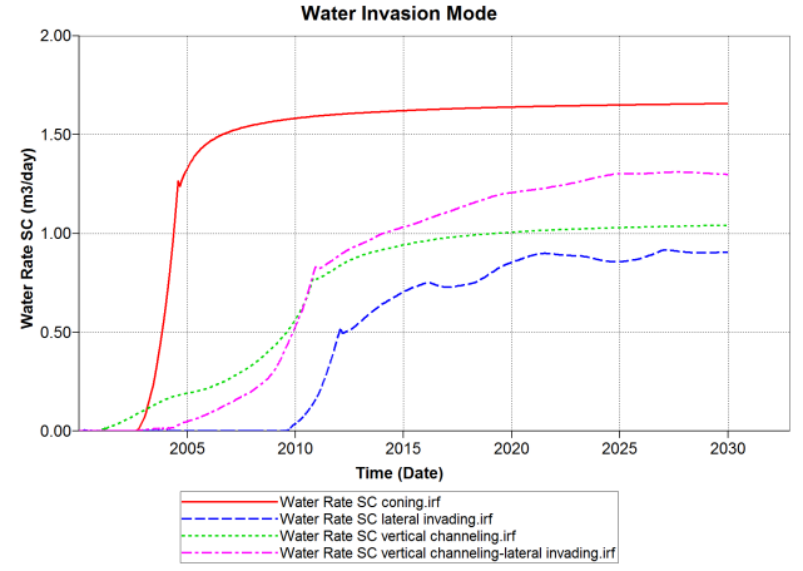

Figure 9. Effect of Water Invasion Type on Water Rate.

\section{Conclusion}

In this paper, an analytical method to obtain the pseudo relative permeability curves for a grid block containing high angle fractures was implemented. The IMEX simulator of CMG was used to investigate the influence of conductive fractures on the water breakthrough phenomenon. Based on the sensitive analysis, the following conclusions can be obtained:

(1) The time of water breakthrough is affected by the fracture angle, the relative distance between high angle fractures and the bottom-water, the fracture length, the production of the well and the water invasion type;

(2) According to the characteristic curve, the water invasion type could be distinguished quickly;

(3)The method lays foundation to further study on the enhanced recovery of the fractured carbonate gas reservoir with water.

\section{Acknowledgement}

This study was supported by National Natural Science Foundation of China (No. 51474226) and (No. 51504264), Major National Research Council of Science and Technology Projects (2016ZX05054011).

\section{Nomenclature}

d aperture, $\mathrm{L}$

$\mathrm{k}$ permeability, $\mathrm{L}^{2}$

$\mathrm{S}$ saturation

$\varphi$ porosity

$\alpha$ contribution to the volume of the total block

$\beta \quad$ contribution to maximum block relative permeability

\section{Subscripts}

b grid block

c connate

m matrix 


$\begin{array}{ll}\text { e } & \text { end point } \\ \mathrm{f} & \text { fracture } \\ \mathrm{g} & \text { gas } \\ \mathrm{w} & \text { water }\end{array}$

\section{References}

[1] Huang, S., Yao, Y.D., et al. Pressure transient analysis of multi-fractured horizontal wells in tight oil reservoirs with consideration of stress sensitivity (ICCESEN 2017), 4-8 October, 2017 Antalya-Turkey. DOI: $10.1007 / \mathrm{s} 12517-018-3639-7$

[2] Xia, C.S., et al. Ways and methods of enhancing recovery in various water-carrying gas reservoirs[J]. Natural gas industry, 2002, 22(z1):73-77

[3] E, Altuncu., S, İriç. Evaluation of Fracture Toughness of Thermal Sprayed and Hard Chrome Coated Aluminium-Zinc Alloy(ICCESEN 2016), 1924 October, 2016 Antalya-Turkey. DOI: 10.12693/APhysPolA.132.926

[4] Peng, X.L., et al. Percolation model and numerical simulation of bottom water gas reservoirs with big fractures[J]. Natural gas industry, 2004, 24(11):116119

[5] Lingen, P., et al. Single Medium Simulation of Reservoirs with Conductive Faults and Fractures. In SPE Middle East Oil Show. Society of Petroleum Engineers, (2001). DOI: 10.2118/68165-MS

[6] Rida, A.G., et al. Single porosity simulation of fractures with low to medium fracture to matrix permeability contrast. In SPE/EAGE Reservoir Characterization and Simulation Conference. Society of Petroleum Engineers, (2001). DOI: 10.2118/125565-MS

[7] Feng, Y. Y. A research on water invasion performance of the gas wells in fractured bottom water reservoir[J]. Natural gas industry, 1998, 18(3):40-44

[8] Mao, X.L., et al. Experimental and numerical simulation on the influence of anisotropic fracture network deformation to shale gas percolation (ICCESEN 2017), 4-8 October, 2017 Antalya-Turkey. DOI: $10.1007 / \mathrm{s} 12517-018-3973-9$ 